\title{
Pre- and early post-partum adiponectin and Interleukin- 1 beta levels in women with and without gestational diabetes
}

\author{
Nikolaos Vitoratos ${ }^{1}$, Georgios Valsamakis ${ }^{1,2}$, George Mastorakos ${ }^{1,2}$, \\ Anastassios Boutsiadis $^{1,2}$, Nikolaos Salakos ${ }^{1}$, Evagelia Kouskouni ${ }^{3}$, George Creatsas ${ }^{1}$ \\ ${ }^{1} 2^{\text {nd }}$ Department of Obstetrics and Gynecology, ${ }^{2}$ Endocrine Unit, ${ }^{3}$ Department of Clinical Biochemistry, "Aretaieion" \\ University Hospital, Athens University Medical School, Athens, Greece
}

\begin{abstract}

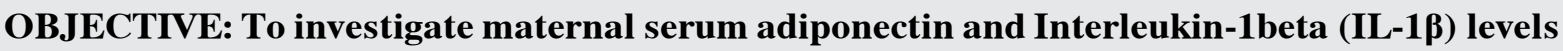
during the pre- and post-partum periods in pregnant women with and without Gestational Diabetes Mellitus (GDM). DESIGN: Thirty control pregnant Caucasian women without GDM and thirty Body Mass Index (BMI) and age-matched Caucasian women with GDM examined in the outpatient clinic between the $24^{\text {th }}$ and $26^{\text {th }}$ week of their pregnancy and on the $3^{\text {rd }}$ day postpartum underwent anthropometry and had serum blood taken. Both groups, were monitored by a dietitian and had comparable weight gain during pregnancy. Birth weight was also measured. RESULTS: At the $3^{\text {rd }}$ day postpartum, compared to the $2^{\text {nd }}$ trimester of pregnancy, women with GDM had lower serum adiponectin levels, lower serum IL-1ß levels and lower Homeostasis Model Assessment for Insulin Resistance (HOMA-IR) values. At the $2^{\text {nd }}$ trimester of pregnancy, women with GDM had lower serum adiponectin levels, higher IL-1ß and higher HOMA-IR values compared to women without GDM. At the $3^{\text {rd }}$ day postpartum, women with GDM had lower serum adiponectin levels, higher IL-1 $\beta$ and higher HOMA-IR values compared to women without GDM. Second trimester serum adiponectin values of women with GDM correlated negatively with birth weight. CONCLUSIONS: Gestational diabetes is a state of insulin resistance associated with altered levels of proinflammatory cytokines, increased IL-1ß and decreased adiponectin values. Both of these alterations might be attributed to placental pathology in pregnancies with GDM.
\end{abstract}

Key words: Adiponectin, Gestational diabetes, IL-1beta, Pre- and early post-partum period

Address for correspondence:

N. Vitoratos MD, Assoc. Professor, $2^{\text {nd }}$ Department of

Ob/Gyn, University of Athens, "Aretaieion" Hospital, 76 Vas.

Sophias Av., 11528 Athens, Greece, Tel: +30 2107484820 ,

Fax: +302107233330

e-mail: nikolasvitoratos@yahoo.gr

Received 08-12-07, Revised 25-04-08, Accepted 20-05-08

\section{INTRODUCTION}

During pregnancy, metabolism undergoes important changes due to the growing fetus and placenta and the increasing metabolic needs of the mother. Pregnancy is associated with alterations in the regulation of glucose metabolism caused by the actions 
of hormones that antagonize the actions of insulin, leading to a state of relative insulin resistance as pregnancy progresses. The decrease of insulin sensitivity during pregnancy parallels feto-placental unit growth and resolves following placenta delivery. Gestational Diabetes Mellitus (GDM) affects roughly $4-8 \%$ of pregnancies. ${ }^{1-3}$ It has been suggested that in GDM there is a partially acquired and partially inherited insulin resistance preceding pregnancy. The partially acquired insulin resistance increases even in women without GDM during the second half of pregnancy and disappears postpartum. ${ }^{4,5}$

Placenta is involved in the process of insulin resistance and fetal growth in both non-diabetic pregnancies and in pregnancies complicated by GDM through human placental lactogen and placental growth hormone production. ${ }^{6}$ Recently, human placenta and adipose tissue have been found to produce various pro-inflammatory factors, including cytokines such as Interleukin-1 beta (IL-1 $\beta$ ) and adiponectin ${ }^{7-9}$ Adiponectin is an adipocytokine with profound insulin sensitizing, anti-inflammatory and antiatherogenic effects. ${ }^{10,11}$ It has also been proposed that adiponectin may play a role in regulating fetal growth in pre-term and term infants of non-diabetic pregnancies. ${ }^{12,13}$ Previous studies have shown hypoadiponectinemia in the $2^{\text {nd }}$ trimester of pregnancies complicated by GDM, as compared to normal pregnancies. ${ }^{14,15}$ In addition, adiponectin is negatively correlated to $2 \mathrm{~h}$ plasma glucose and insulin concentrations of the oral glucose tolerance test in pregnancies with and without $\mathrm{GDM}^{16}$ and positively to insulin secretion sensitivity index only in pregnancies with GDM. ${ }^{17}$ Studies show no significant change of maternal serum adiponectin during all trimesters of normal pregnancy, ${ }^{18,19}$ while others show progressive decrease. ${ }^{20,21}$ Furthermore, IL-1 $\beta$, a pro-inflammatory cytokine, has been suggested as being involved in the process of glucotoxicity and impaired insulin secretion in diabetes mellitus, while it is increased at pre-term labor in non-diabetic pregnancy and in atherogenesis in patients with coronary heart disease. ${ }^{22-26}$ To date, there are no studies comparing maternal serum levels of IL-1 $\beta$ between pregnancies with and without GDM.

During pregnancy, adipocytokines are produced not only by the adipose tissue but also by the placenta; it is thus not known to what extent the latter is involved in the process of insulin resistance in pregnancies with and without GDM. Recent data showed a possible dysregulation of human placenta function in pregnancies with GDM, ${ }^{27}$ while it is known that in these pregnancies insulin resistance disappears at 8-12 weeks post-partum. ${ }^{28,29}$ There are as yet no studies showing the serum concentrations of IL- $1 \beta$ and adiponectin immediately after delivery in pregnancies with GDM. The aim of our study was to investigate the possible interplay of maternal serum adiponectin and IL-1 $\beta$ levels in pre- $\left(2^{\text {nd }}\right.$ trimester $)$ and post-partum ( $3^{\text {rd }}$ day after delivery) periods, as well as their effect on birth weight in Body Mass Index (BMI) matched pregnant women with and without GDM.

\section{MATERIALS AND METHODS}

\section{Patient recruitment}

The study had the approval of the local Ethics Committee and written informed consent was obtained from all subjects. Thirty control primigravidae pregnant Caucasian women [with mean BMI $( \pm$ SD) of $26.2 \pm 3.6 \mathrm{~kg} / \mathrm{m}^{2}$ and aged $31.2 \pm 4.5$ years] and thirty BMI and age matched primigravidae pregnant Caucasian women with Gestational Diabetes [mean BMI $\left( \pm\right.$ SD) of $26.6 \pm 4.9 \mathrm{~kg} / \mathrm{m}^{2}$ and aged $32.2 \pm$ 3.4 years] recruited randomly from the Obstetrics and Gynecology outpatient clinic of the Aretaieion University Hospital. GDM was diagnosed after a $75 \mathrm{gr}$ Oral Glucose Tolerance Test (OGTT), carried out between the $24^{\text {th }}$ and $26^{\text {th }}$ week of pregnancy using the WHO criteria. ${ }^{30,31}$ During pregnancy and labor, no intrauterine infection, preeclampsia, pregnancy-induced hypertension or any other pregnancy complication occurred and all studied mothers were non-smokers.

\section{Protocol}

The recruited pregnant women were seen in the outpatient clinic between the $24^{\text {th }}$ and $26^{\text {th }}$ week of their pregnancy and on the $3^{\text {rd }}$ day postpartum. Throughout pregnancy, the women were followed up by a dietitian. At each visit they were submitted to the following clinical and biochemical examination:

\section{i) Anthropometric measurements}

All measurements were carried out by a single observer. Maternal weight was measured in kilograms 
to the nearest $0.1 \mathrm{~kg}$ on a beam balance. Height in meters was measured to the nearest $\mathrm{mm}$ using a stadiometer and the BMI in kilograms per square meter was calculated. Postpartum BMI was not significantly different between the two groups. Women with and without GDM had BMI of 30.3 \pm 4.3 and 29.9 \pm 4.1 , respectively $(p=0.450)$. The weight of the neonate at birth was taken by a single paediatrician.

\section{ii) Biochemical assays}

After an overnight fast, a 75 gr OGTT was performed and blood samples were drawn for measurement of glucose, insulin, IL-1 $\beta$, adiponectin, estradiol and progesterone at the time-points of $0 \mathrm{~min}$ and at 5 , $15,30,60,90$ and $120 \mathrm{~min}$ for measurement of insulin and glucose. Blood samples were stored at $-70^{\circ} \mathrm{C}$. Insulin levels were determined using the Medgenic immunoenzymetric assay (Biosource-Europe SA, 1400 Nivelles, Belgium). Estradiol and progesterone levels were determined using the analytical performance of new estradiol and progesterone assays performed on the Bayer Immuno 1 system (Bayer Corp. Business Group Diagnostics, Tarrytown NY 10591-5097, USA). Inter- and intra-assay coefficients of variation for estradiol were $6.4 \%$ and $5.8 \%$, respectively, and for progesterone $<7.7 \%$ and $8.2 \%$, respectively. IL-1 $\beta$ was determined using an ELISA human kit (purchased from R\&D Systems Inc, Minneapolis, Minnesota, 55413 , USA). IL- $1 \beta$ sensitivity was less than $1 \mathrm{pg} / \mathrm{mL}$. Inter- and intra-assay coefficients of variation were $8.4 \%$ and $8.5 \%$, respectively. Serum adiponectin was determined using an ELISA kit (purchased from Chemicon International Inc., Temecula, CA, USA). Adiponectin sensitivity was $0.1 \mathrm{ng} / \mathrm{mL}$ with a range of detection from 0.230 to $15.0 \mathrm{ng} / \mathrm{mL}$. Inter- and intra-assay coefficients of variation were $9.8 \%$ (at $7.5 \mathrm{ng} / \mathrm{mL}$ ) and $8.4 \%$ (at $3.7 \mathrm{ng} / \mathrm{mL}$ ), respectively. Insulin resistance was derived from fasting glucose and insulin levels using the homeostasis mathematical model assessment (HOMA-IR) [insulin at baseline $(\mathrm{pmol} / \mathrm{L}) \times$ glucose at baseline $(\mathrm{mmol} / \mathrm{L})] / 135 .{ }^{32}$

\section{Statistical analysis}

Data are described as mean \pm SD (median and interquartile range for data not normally distributed). The Mann Whitney U test was used to test for differences between the two groups on a continuous measure in instances of non-normally distributed variables. To test for changes of variables pre- and post-partum in a group of patients, the Wilcoxon signed rank test was used for non-normally distributed variables. To test the associations between different variables, the Spearmann correlation analysis was performed. A p-value of $<0.05$ was considered to be significant. The SPSS statistical software No 12 was used for statistical analysis (SPSS Inc., Chicago, IL, 60606, USA). ${ }^{33}$

\section{RESULTS}

\section{Comparisons of adiponectin and $I L-1 \beta$ levels and HOMA-IR values within groups of pregnant women with and without GDM at pre- and post-partum}

At the $3^{\text {rd }}$ day postpartum compared to $2^{\text {nd }}$ trimester of pregnancy, women with GDM had significantly lower serum adiponectin levels [1.78 (1.06-3.48) vs 4.88 (3.46-6.59) mg/L, respectively, $\mathrm{p}=0.002$ ] (Figure 1$)$; significantly lower serum IL-1 $\beta$ levels $[0.58$ (0.49-0.98) vs $1.39(0.73-1.58) \mathrm{ng} / \mathrm{mL}$, respectively, $\mathrm{p}=0.003$ ] (Figure 2); and significantly lower HOMAIR values [2.3 (1.51-4.6) vs 3.31 (2.22-5.8), respectively, $\mathrm{p}=0.027]$.

At the $3^{\text {rd }}$ day postpartum compared to $2^{\text {nd }}$ trimester

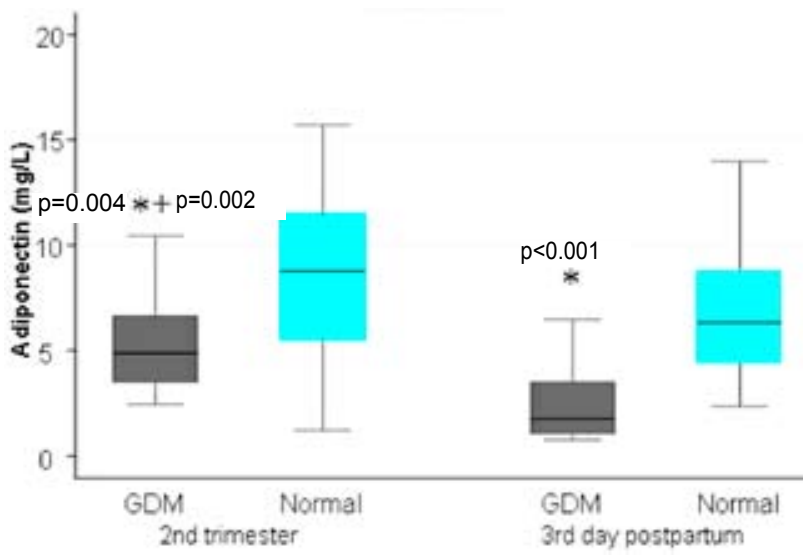

Figure 1. Maternal adiponectin levels at $2^{\text {nd }}$ trimester of pregnancy and $3^{\text {rd }}$ day postpartum in women with and without (normal) GDM expressed as box-plot of $25^{\text {th }}$ (lower limit) to $75^{\text {th }}$ (upper limit) percentile. The horizontal line in the box-plot stands for median. The asterisk indicates a statistically significant difference from normal women at the same time-point of observation; the cross indicates a statistically significant difference between the two time-points of observation of the same group of women. 


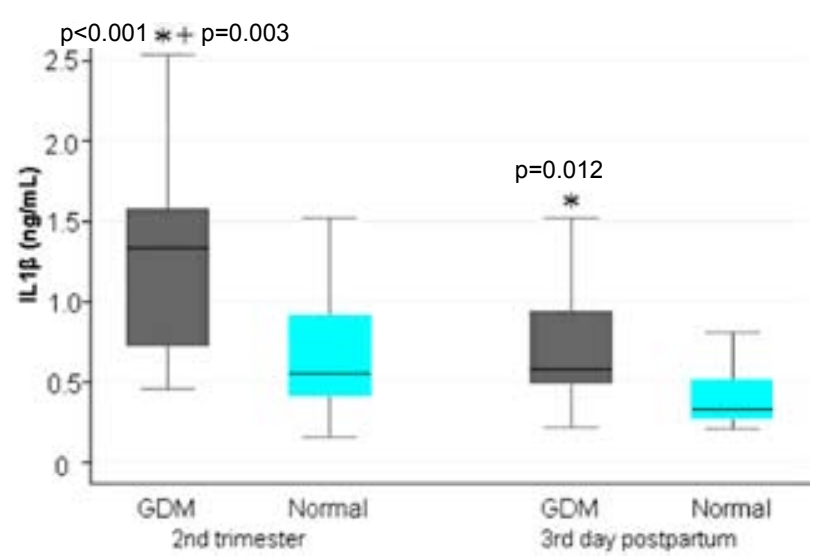

Figure 2. Maternal IL-1 $\beta$ levels at $2^{\text {nd }}$ trimester of pregnancy and $3^{\text {rd }}$ day postpartum in women with and without (normal) GDM expressed as box-plot of $25^{\text {th }}$ (lower limit) to $75^{\text {th }}$ (upper limit) percentile. The horizontal line in the box-plot stands for median. The asterisk indicates a statistically significant difference from normal women at the same time-point of observation; the cross indicates a statistically significant difference between the two time-points of observation of the same group of women.

of pregnancy, in the women with normal pregnancies there was a downward trend of serum adiponectin levels [6.35 (4.42-8.79) vs 8.79 (5.47-11.5) $\mathrm{mg} / \mathrm{L}$, respectively, $\mathrm{p}=0.175]$ (Figure 1), a downward trend of serum IL-1 $\beta$ levels $[0.33$ (0.27-0.51) vs 0.55 (0.42-0.91) $\mathrm{ng} / \mathrm{mL}$, respectively, $\mathrm{p}=0.070$ ] (Figure 2 ) and no significant change in HOMA IR values [1.7 (1.34-3.64) vs 1.91 (1.61-3.93), respectively, $\mathrm{p}=0.210]$.

Estradiol and progesterone levels did not differ between pregnant women with and without GDM at all time-points studied.

\section{Comparisons of adiponectin and $I L-1 \beta$ levels and HOMA-IR values between groups of pregnant women with and without GDM at pre- and post-partum periods}

At the 2nd trimester of pregnancy, women with GDM had significantly lower serum adiponectin levels compared to normal pregnancies [median 4.88 (3.466.59) vs 8.79 (5.47-11.5) $\mathrm{mg} / \mathrm{L}$, respectively, $\mathrm{p}=0.004$ ] (Figure 1); significantly higher serum IL-1 $\beta$ levels [median 1.39 (0.73-1.58) vs $0.55(0.42-0.91) \mathrm{ng} / \mathrm{mL}$, respectively, $\mathrm{p}<0.001$ ] (Figure 2); and significantly higher HOMA-IR values [median 3.31 (2.22-5.8) vs 1.91 (1.61-3.93), respectively, $p=0.003$ ].

At the 3rd day postpartum, women with GDM had significantly lower serum adiponectin levels compared to normal pregnancies [median $\left(25^{\text {th }}-75^{\text {th }}\right.$ percentile $)$, 1.77 (1.06-3.48) vs 6.35 (4.42-8.79) $\mathrm{mg} / \mathrm{L}$, respectively, $\mathrm{p}<0.001$ ] (Figure 1); significantly higher IL-1 $\beta$ levels [0.58 (0.49-0.98) vs $0.33(0.27-0.51) \mathrm{ng} / \mathrm{mL}$, respectively, $\mathrm{p}=0.012$ ] (Figure 2); and significantly higher HOMA-IR values [2.3 (1.51-4.6) vs 1.7 (1.34-3.64), respectively, $\mathrm{p}=0.032]$.

There was a trend towards higher birth weight in GDM compared to normal pregnancies [2977 (2450-3450) vs 3246 (2910-3568) grams, respectively, $\mathrm{p}=0.230]$.

\section{Correlations of adiponectin and IL-1ß levels with birth weight}

Serum adiponectin in the $2^{\text {nd }}$ trimester of GDM pregnancies correlated negatively with birth weight $(\mathrm{p}=0.005, \mathrm{r}=-0.6)$. There was no statistically significant correlation between serum adiponectin levels and birth weight in normal pregnancies $(\mathrm{p}=0.310)$. There were no statistically significant correlations between adiponectin and IL- $1 \beta$ levels at the studied time-points nor between pre- to post-partum change $(\Delta)$ of adiponectin and IL-1 $\beta$ levels in GDM pregnancies $(\mathrm{p}=0.421)$.

\section{DISCUSSION}

In this prospective study, we investigated the pre- and post-partum serum levels of adiponectin and IL-1 $\beta$ in parallel with insulin resistance in BMI matched pregnancies with and without GDM. Pregancies with GDM had a weight gain similar to pregnancies without GDM. Placenta is an endocrine organ producing hormones and cytokines, involved in the process of pregnancy and labor induction. ${ }^{25,34}$ Previous studies have shown placental production of cytokines such as IL-1 $\beta$ and adiponectin in normal pregnancy. ${ }^{8,9,27}$

In the present study, pregnant women with GDM showed at second trimester (a period of insulin resistance development) higher insulin resistance compared to BMI- and age-matched pregnant women without GDM. Furthermore, pregnant women with GDM presented at $2^{\text {nd }}$ trimester lower serum adiponectin and higher IL-1 $\beta$ levels than women without GDM. Thus, it seems that GDM is associated with increased 
pro-inflammatory cytokine production such as IL$1 \beta$ and lower production of adiponectin, an insulin sensitivity-related adipocytokine. These findings are in accordance with recent studies reporting that pregnancies with GDM are associated with low adiponectin levels during the $2^{\text {nd }}$ trimester ${ }^{35-37}$ and increased levels of TNF $\alpha$, IL-6 and high sensitivity (hs) CRP, all indicators of a pro-inflammatory status. ${ }^{35,37,38} \mathrm{In}$ the present study, it is shown that IL- $1 \beta$ levels follow a pattern similar to the other pro-inflammatory cytokines in pregnancies with GDM. The source of maternal pro-inflammatory cytokines during pregnancy is not entirely clear.

In the present study, serum IL-1 $\beta$ and adiponectin levels significantly decreased from the $2^{\text {nd }}$ trimester of pregnancy to the $3^{\text {rd }}$ postpartum day in pregnancies with GDM. It is possible that this postpartum reduction of adiponectin levels reflects the insulin resistance-associated adiponectin decrease during pregnancy in GDM. In normal pregnancies, a decreasing trend in adiponectin values was observed. Recently, it was shown that serum hsCRP decreased while TNFalpha and adiponectin levels remained unchanged four months postpartum in pregnancies with and without GDM. ${ }^{39}$ On the other hand, other investigators found hypoadiponectinemia during the $3^{\text {rd }}$ and $4^{\text {th }}$ day postpartum in normal lactating women. ${ }^{40,18}$ It has been suggested that this hypoadiponectinemia might be due to the suppression of adipocyte-produced adiponectin by prolactin. ${ }^{40,41}$ Otherwise, the significant decrease of the studied cytokines during the early postpartum period in pregnancies with GDM in this study could be explained by the withdrawal of cytokines produced by the placenta during pregnancy. ${ }^{8,9,27}$ The role of the placenta in the production of adiponectin and IL- $1 \beta$ during pregnancy is further reinforced by the non-significant but clear downward trend of these cytokines during third day postpartum found in pregnancies without GDM. The $3^{\text {rd }}$ day postpartum is a period in which the effect of the placenta on maternal metabolism regarding insulin resistance has just been removed and lactation is started. It is possible that in pregnancies with GDM there is a more prominent placental adiponectin production, possibly to compensate for the increased insulin resistance, an effect that is modest in normal pregnancies..$^{19}$ Indeed, it has been suggested that there is an alteration of placental function in pregnancies with GDM. ${ }^{27}$

Although not statistically significant, this study disclosed that pregnancies with GDM are associated with higher birth weights than pregnancies without GDM. Furthermore, the present study revealed maternal serum adiponectin levels at second trimester to be negatively correlated with birth weight in pregnancies with GDM. This is in accordance with previous studies indicating a negative correlation of macrosomia and/or birth weight with maternal adiponectin levels in pregnancies with GDM. ${ }^{16,37}$ By contrast, in pregnancies without GDM a positive correlation between fetal adiponectin and fetus-toplacenta weight ratio has been shown, suggesting a possible role of adiponectin in fetal growth. ${ }^{12}$ Cseh et al have found an inverse relationship between the newborn's body length, head circumference and body weight and maternal TNF-alpha, leptin and C-peptide levels in pregnancies with GDM. ${ }^{42}$ In our study, no correlation was found between maternal IL-1 $\beta$ and birth weight.

In conclusion, pregnancy with GDM is a state of insulin resistance associated with increased IL-1 $\beta$ and decreased adiponectin values. Both of these alterations might be attributed to placenta involvement in pregnancies with GDM.

\section{ACKNOWLEDGEMENTS}

This work has been supported by a research grant from the Ethnikon and Kapodistriakon University of Athens.

\section{REFERENCES}

1. Buchanan TA, Kjos SL, 1999 Gestational diabetes: risk or myth? J Clin Endocrinol Metab 84: 1854-1857.

2. Hadden DR, 1985 Geographic, ethnic and racial variations in the incidence of gestational diabetes mellitus. Diabetes 34: 8-12.

3. Beischer NA, Wein P, Sheedy MT, et al, 1996 Identification and treatment of women with hyperglycemia diagnosed during pregnancy can significantly reduce perinatal mortality rates. Aust NZ Obstet Gynaecol 36: 239-247.

4. Buchanan TA, Catalano PM, 1995 The pathogenesis of GDM: implication for diabetes after pregnancy. Diabetes Rev 3: 584-601. 
5. Sivan E, Chen X, Homko CJ, et al, 1997 Longitudinal study of carbohydrate metabolism in healthy obese pregnant women. Diabetes Care 20: 1470-1475.

6. Day IN, Chen XH, Gaunt TR, et al, 2004 Late life metabolic syndrome, early growth, and common polymorphism in the growth hormone and placental lactogen gene cluster. J Clin Endocrinol Metab 89: 5569-5576.

7. Lappas M, Permezel M, Rice GE, 2005 Leptin and adiponectin stimulate the release of proinflammatory cytokines and prostaglandins from human placenta and maternal adipose tissue via nuclear factor- $x \mathrm{~B}$, peroxismal proliferators-activated receptor- $\gamma$ and extracellularly regulated kinase 1/2. Endocrinology 146: 3334-3342.

8. Steinborn A, von Gall C, Hildenbrand R, et al, 1998 Identification of placental cytokine-producing cells in term and preterm labor. Obstet Gynecol 91: 329-335.

9. Chen J, Tan B, Karteris E, et al, 2006 Secretion of adiponectin by human placenta: differential modulation of adiponectin and its receptors by cytokines. Diabetologia 49: 1292-1302.

10. Yang WS, Lee WJ, Funahashi T, et al, 2001 Weight reduction increases plasma levels of an adipose-derived anti-inflammatory protein adiponectin. J Clin Endocrinol Metab 86: 3815-3819.

11. Ouchi N, Kihara S, Arita Y, et al, 2000 Adiponectin, an adipocyte-derived plasma protein, inhibits endothelial NF-kappaB signaling through a cAMP-dependent pathway. Circulation 102: 1296-1301.

12. Kadowaki K, Waguri M, Nakanishi I, et al, 2006 Adiponectin concentration in umbilical cord serum is positively associated with the weight ratio of fetus to placenta. J Clin Endocrinol Metab 91: 5090-5094.

13. Kajantie E, Hytinannti T, Hovi P, et al, 2004 Cord plasma adiponectin: a 20 -fold rise between 24 weeks gestation and term. J Clin Endocrinol Metab 89: 4031-4036.

14. Worda C, Leipold H, Gruber C, et al, 2004 Decreased plasma adiponectin concentrations in women with gestational diabetes. Am J Obstet Gynecol 191: 2120-2124.

15. Cortelazzi D, Corbetta S, Ronzoni S, et al, 2007 A. Maternal and fetal resistin and adiponectin concentrations in normal and complicated pregnancies. Clin Endocrinol 66: 447-453.

16. Tsai PJ, Yu CH, Hsu SP, et al, 2005 Maternal plasma adiponectin concentrations at 24 to 31 weeks of gestation: negative association with gestational diabetes mellitus. Nutrition 21: 1095-1099.

17. Retnakaran R, Hanley AJ, Raif N, et al, 2005 Adiponectin and beta cell function in gestational diabetes: pathophysiological implications. Diabetologia 48: 993-1001.

18. Mazaki-Tovi S, Kanety H, Pariente C, et al, 2007 Maternal serum adiponectin levels during human pregnancy. J Perinatol 27: 77-81.

19. Mastorakos G, Valsamakis G, Papatheodorou D, et al, 2007 The role of adipocytokines in normal pregnancy: visfatin levels in early pregnancy predict insulin sensitivity. Clin Chem 53: 1-10.
20. Fuglsang J, Skjaerbaeck C, Frystyck J, et al, 2006 A longitudinal study of serum adiponectin during normal pregnancy. BJOG 113: 110-113.

21. Catalano PM, Hoegh M, Minium J, et al, 2006 Adiponectin in human pregnancy: implications for regulation of glucose and lipid metabolism. Diabetologia 49: 16771685 .

22. Maedler K, Schumann DM, Sauter N, et al, 2006 Low concentration of interleukin-1 beta induces FLICE-inhibitory protein-mediated beta cell proliferation in human pancreatic islets. Diabetes 55: 2713-2722.

23. Maedler K, Sergeev P, Ris F, et al, 2002 Glucose-induced beta cell production of IL-1beta contributes to glucotoxicity in human pancreatic islets. J Clin Invest 110: 851-860.

24. Steinborn A, Gunes H, Roddiger S, et al, 1996 Elevated placental cytokine release, a process associated with preterm labor in the absence of intrauterine infection. Obstet Gynecol 88: 534-539.

25. Vitoratos N, Mastorakos G, Kountouris A, et al, 2007 Positive association of serum interleukin-1beta and $\mathrm{CRH}$ levels in women with pre-term labor. J Endocrinol Invest 30: 35-40.

26. Latkovskis G, Licis N, Kalnins U, 2004 C-reactive protein levels and common polymorphisms of the interleukin-1 gene cluster and interleukin-6 gene in patients with coronary heart disease. Eur J Immunogenet 31: 207-213.

27. Lappas M, Yee K, Permezel M, et al, 2005 Release and regulation of leptin and adiponectin from human placenta, fetal membranes and maternal adipose tissue and skeletal muscle from normal and gestational diabetes mellitus complicated pregnancies. J Endocrinol 186: 457-465.

28. Homko C, Sivan E, Chen X, et al, 2007 Insulin secretion during and after pregnancy in patients with gestational diabetes mellitus. J Clin Endocrinol Metab 86: 568573.

29. Kautzky-Willer A, Prager R, Waldhausl W, et al, 1997 Pronounced insulin resistance and inadequate beta-cell secretion characterize lean gestational diabetes during and after pregnancy. Diabetes Care 20: 1717-1723.

30. World Health Organisation Study Group on Diabetes Mellitus. Technical Report Series 727. WHO Geneva 1985

31. Diabetes Pregnancy Study Group 1989 A prospective multicentre study to determine the influence of pregnancy upon the $75 \mathrm{gr}$ oral glucose tolerance test (OGTT). In: Sutherland HN, Stowers JM, Pearson DWM (eds), Carbohydrate Metabolism in Pregnancy and the Newborn IV. Springer-Verlag, London; pp, 209-226.

32. Matthews DR, Hosker JP, Rudenski AS, et al, 1985 Homeostasis model assessment: insulin resistance and $\beta$-cell function from fasting plasma glucose and insulin concentrations in man. Diabetologia 28: 412-419.

33. SPSS. SPSS for Windows. 12.0. Chicago: SPSS Inc., 1999.

34. Ticconi C, Belmonte A, Piccione E, et al, 2006 Feto- 
placental communication system with the myometrium in pregnancy and parturition: the role of hormones, neurohormones, inflammatory mediators, and locally active factors. J Matern Fetal Neonatal Med 19: 125-133.

35. Kinalski M, Telejko B, Kuzmincki M, et al, 2005 Tumor necrosis factor alpha system and plasma adiponectin concentration in women with gestational diabetes. Horm Metab Res 37: 450-454.

36. Retnakaran R, Connelly RW, Maguire G, et al, 2007 Decreased high molecular weight adiponectin in gestational diabetes: implications for the pathophysiology of type 2 diabetes. Diabet Med 24: 245-252.

37. Ategbo JM, Grissa O, Yessoufou A, et al, 2006 Modulation of adipokines and cytokines in gestational diabetes and macrosomia. J Clin Endocrinol Metab 91: 4137-4143.

38. Bo S, Signorile A, Menato G, et al, 2005 C-reactive protein and tumor necrosis factor alpha in gestational hyperglycemia. J Endocrinol Invest 28: 779-786.
39. McLachlan KA, O Neal D, Jenkins A, et al, 2006 Do Adiponectin, TNFalpha, leptin and CRP relate to insulin resistance in pregnancy? Studies in women with and without gestational diabetes during and after pregnancy. Diab Metab Res Rev 22: 131-138.

40. Asai-Sato M, Okamoto M, Endo M, et al, 2006 Hypoadiponectinemia in lean lactating women: Prolactin inhibits adiponectin secretion from human adipocytes. Endocr $\mathbf{J}$ 53: 555-562.

41. Ben-Jonathan N, Hugo ER, Brandenburg TD, et al, 2006 Focus on prolactin as a metabolic hormone. Trends Endocrinol Metab 17: 110-116.

42. Cseh K, Baranyi E, Melczer Z, et al, 2002 The pathophysiological influence of leptin and the tumor necrosis factor system on maternal insulin resistance: negative correlation with anthropometric parameters of neonates on gestational diabetes. Gynecol Endocrinol 16: 453-460. 